\title{
Mycobacterium tuberculosis latency-associated antigen Rv1733c SLP improves the accuracy of differential diagnosis of active tuberculosis and latent tuberculosis infection
}

\section{Lifan Zhang}

Peking Union Medical College Hospital

\section{Huimin Ma}

PUMCH: Peking Union Medical College Hospital

\section{Shijun Wan}

Peking Union Medical College Hospital

\section{Yueqiu Zhang}

Peking Union Medical College Hospital

\section{Mengqiu Gao}

Beijing Chest Hospital

Xiaoqing Liu ( $\square$ liuxq@pumch.cn )

Capital Medical University/Beijing https://orcid.org/0000-0001-8162-0903

\section{Research Article}

Keywords: Mycobacterium tuberculosis latency associated antigens, Active tuberculosis, Latent tuberculosis infection, Differential diagnosis

Posted Date: December 7th, 2020

DOI: https://doi.org/10.21203/rs.3.rs-121733/v1

License: (c) (1) This work is licensed under a Creative Commons Attribution 4.0 International License. Read Full License

Version of Record: A version of this preprint was published at Chinese Medical Journal on November 19th, 2021. See the published version at https://doi.org/10.1097/CM9.0000000000001858. 


\section{Abstract \\ Background}

The differential diagnosis of active tuberculosis(ATB) and latent tuberculosis infection(LTBI) is still challenging. The objective of the study was to evaluate the accuracy of the novel M. tuberculosis latencyassociated antigens Rv1733c and Rv1733c SLP for differentiating ATB from LTBI.

\section{Methods}

A case-control study was designed to enroll pathogen-confirmed ATB cases admitted to the Peking Union Medical College Hospital and Beijing Chest Hospital, whereas those with LTBI were denoted as the control group. The Fluorescence-Immunospot (FluoroSpot) assay was used to detect the frequencies of IL-2-, IFN$\mathrm{Y}$-secreting $\mathrm{T}$ cells stimulated by the $\mathrm{M}$. tuberculosis latency-associated antigens Rv1733c and Rv1733c SLP. The combination of the ESAT-6/CFP-10-Fluorospot test was evaluated with regard to the sensitivity, specificity, predictive value and likelihood ratio for the differential diagnosis of ATB and LTBI.

\section{Results}

A total of 20 pathogens-confirmed TB and 28 LTBI cases were included. The sensitivity and specificity of ESAT-6/CFP-10-FluoroSpot for the differential diagnosis of ATB and LTBI were 95\% (95\% Cl, 75.13$99.87 \%)$ and $82.14 \%(95 \% \mathrm{Cl}, 63.11-93.94 \%)$, respectively. Following stimulation with Rv1733c and Rv1733c SLP, the maximum AUROC was $0.711(95 \% \mathrm{Cl}, 0.566-0.856)$ as determined by the ROC curve, which was used to assess the frequency of single IL-2-secreting T cells stimulated by Rv1733c SLP. The cutoff value of 0 SFCs $/ 2.5 \times 105$ PBMCs was used for the analysis. The frequency, sensitivity and specificity of Rv1733c SLP for differentiating ATB and LTBI were 75\% (95\% Cl, 50.90-91.34\%) and $60.71 \%$ (95\% Cl, 40.58-78.50\%), respectively. The ESAT-6/cfp-10-fluorospot was combined with the frequency of single IL-2-secreting T cells, which were stimulated by Rv1733c SLP for the differential diagnosis of ATB and LTBI. This resulted in an increased sensitivity and specificity to $100 \%(95 \% \mathrm{Cl}$, $83.16-100.00 \%)$, as determined by the parallel test and to $92.86 \%(95 \% \mathrm{Cl}, 71.77-97.73 \%)$ as determined by the serial test, respectively.

\section{Conclusions}

Rv1733c SLP has the potential to be used as a candidate antigen for T cell-based tuberculosis diagnostic tests, in combination with ESAT- 6 and CFP-10, to differentiate between ATB and LTBI diagnosis.

\section{Background}


At present, tuberculosis (TB) is one of the most important public health problems in the world. TB is caused by Mycobacterium tuberculosis (MTB) and remains one of the leading cause of death from a single infectious agent [1]. According to the 2020 Global Tuberculosis Report, a total of 10 million new cases of tuberculosis were discovered in 2019 worldwide. China exhibits the third largest number of TB patients in the world, accounting for $8.4 \%$ of the world's TB patients, after India (26\%) and Indonesia (8.5\%) [1].

Traditional methods for TB diagnosis have several disadvantages. The sensitivity of smear antacid staining detection is poor and the bacterial culture is often time-consuming. The Xpert MTB/RIF results can be obtained within hours, whereas $30-50 \%$ of TB patients are not applicable due to the lack of qualified samples. Previous research studies conducted by our team indicated that more than two-thirds of TB diagnosis in PUMCH were based on clinical criteria rather than being confirmed by etiology[2]. Therefore, new methods are urgently required to aid clinicians make differential diagnosis in patients suspected of TB that are unable to provide etiological evidence.

In 1991, Behr et al. [3] analyzed and compared the whole genome of H37Rv of MTB and BCG and found the difference region (RD) between MTB and BCG, named RD1-16. RD1 region does not exist in all BCG and in the majority of other mycobacterial genomes. Two secretory proteins, ESAT- 6 and CFP-10, encoded by RD1-Rv3875 and RD1-Rv3874, have been shown to exhibit optimal immunogenicity and can be used as MTB specific antigens to stimulate the immune response. The aforementioned findings lay the foundation for the use of IGRAs. In the past 20 years, IGRAs have been widely used in the diagnosis of tuberculosis infection, especially in the immunosuppressive population and areas where BCG vaccines were included in the neonatal vaccination plan. The accuracy of IGRAs in the diagnosis of tuberculosis infection is better than that of the TST test [4-6]. However, IGRAs fail to distinguish ATB from LTBI. This research topic is considered a hotspot and of great clinical significance for the differential diagnosis of ATB and LTBI. The addition of new antigens in the assay and the detection of new cytokines aim to improve the sensitivity and specificity of the already existing diagnostic methods.

Mycobacterium tuberculosis latency-associated antigens mainly include proteins related to hypoxia, nutritional deficiency, resuscitation and reactivation of MTB. Voskuil et al. [7]constructed the in vitro hypoxia model of MTB and named the gene set which was significantly upregulated as dormancy survival regulon (DosR). Rv1733c, a conservative membrane protein encoded by DosR, is the most commonly recognized latency antigen in M. tuberculosis-exposed household contacts from South Africa, Gambia, and Uganda [8]. Various human cohort studies indicated preferential recognition of DosRencoded proteins, such as Rv1733c, by T cells from subjects with LTBI and to a lesser extent by TB patients $[8,9]$. High levels of IFN- $\gamma$ can be detected in LTBI following stimulation with the Rv1733c antigen [10]. Following inoculation of mice with recombinant BCG expressing Rv1733c, significantly higher IFN-y levels were detected in the supernatant of mouse spleen cells, which could enhance the long-term protection against MTB [11]. The combination of synthetic long peptides (SLPs) and adjuvants has been mainly studied for therapeutic cancer vaccines, yet not for TB and was shown to induce efficient antitumor immunity. The improved long-term protection against MTB in mice was achieved by BCG 
expressing Rv1733c [11]. Based on this evidence Mariateresa Coppola [12] investigated the potential of Rv1733c SLP as vaccines for TB. They synthesized the Rv1733c SLP, which caused a stronger immune response and produced higher levels of IFN-y than those of Rv1733c in LTBI. It is worth assessing whether Rv1733c SLP can be used as an alternative antigen for the differential diagnosis of ATB and LTBI.

As an improvement of the traditional Enzyme-Linked Immunospot (ELISPOT) Assay, the Fluorospot assay is capable of simultaneously detecting multiple cytokines by utilizing fluorochrome-conjugated antibodies at the single cell level without causing spotted color mixing. Previous studies conducted by our research team confirmed that the IFN- $\mathrm{Y} / \mathrm{IL}-2$ FluoroSpot assay stimulated by ESAT- 6 and CFP-10 was conducive for the differential diagnosis of ATB and LTBI $[13,14]$. When the cut-off values of frequency and the proportion of single IFN- - -secreting T cells were set to 25 SFCs $/ 2.5 \times 105$ PBMCs and $43.6 \%$ respectively, the accuracy of the differential diagnosis was optimal[14]. However, significant improvements can also be made for the clinical application of this method. The purpose of the present study was to determine whether the application of Mycobacterium tuberculosis latency-associated antigen Rv1733c/Rv1733c SLP, based on ESAT-6 / CFP-10-fluorospot, could improve the accuracy of the differential diagnosis of ATB and LTBI.

\section{Methods}

Study design and subjects

A case-control study was designed to enroll pathogen-confirmed ATB cases admitted to the Peking Union Medical College Hospital and Beijing Chest Hospital from January to December 2017, whereas those with LTBI were denoted as the control group. The inclusion criteria for the ATB group were the following: 1) age range of 18-75 years; 2) Subjects with active tuberculosis manifestations, such as fever, cough and chest pain; 3) Positive results of smear acid-fast stain or culture of MTB, MTB nucleic acid or Xpert MTB/RIF; 4) Lack of anti-TB treatment. The inclusion criteria for the LTBI group were the following: 1) age range of 18-75 years; 2) Absence of clinical manifestations of active TB; 3) No medical history of TB, no manifestations of previous TB in the chest radiogram; 4) Positive results of T-SPOT for TB. The following exclusion criteria were used: 1) pregnancy or lactation; 2) HIV positive. All subjects participated in the study voluntarily and signed the relevant informed consent form.

Rv1733c peptide synthesis:

Jill Biochemical (Shanghai) Co., Ltd. synthesized and purified M. tuberculosis hypoxia-associated latency antigens Rv1733c and Rv1733c SLP. Rv1733c contains 19 peptides and each peptide has 20 amino acids with both ends overlapping on 10 amino acids. Rv1733c SLP contains 19 peptides and each peptide has 28 amino acids with 14 amino acids overlapping at both ends. Homogeneity and purity were confirmed by analytical high-pressure liquid chromatography (HPLC) and by mass spectrometry. The purity of all peptides was $\geq 80 \%$. 
A total of $4 \mathrm{ml}$ of peripheral blood, anticoagulated with gradient centrifugation was obtained within $4 \mathrm{~h}$. The AIM-V medium (Gibco ${ }^{\text {TM }}$ AIM V Medium liquid, Invitrogen, USA) was used to prepare a cell suspension with a concentration of $2.5 \times 10^{6} \mathrm{PBMCs} / \mathrm{ml}$. 96-well plates were pre-coated with monoclonal antibodies against interferon $\gamma$ (IFN- $\gamma$ ) and interleukin 2 (IL-2). A total of $50 \mu \mathrm{l}$ AIM-V cell culture medium was used to the single wells as a negative control and $5 \mu \mathrm{g} / \mathrm{ml}$ phytohemagglutinin (PHA) were added to multiple wells as a positive control; ESAT-6 peptide, CFP-10 peptide, Rv1733c peptide and Rv1733c SLP were added to multiple wells, respectively. A total of $2.5 \times 10^{5}$ PBMCs and anti-CD28 $(0.5 \mu \mathrm{g} / \mathrm{ml}, \mathrm{AID}, \mathrm{Straßberg}$, Germany) were added to each well. The plates were incubated for $16-20 \mathrm{~h}$ at $37^{\circ} \mathrm{C}$ in $5 \% \mathrm{CO}_{2}$. The plates were incubated with IFN-y-FITC and IL-2-biotin tagged with fluorescein and further incubated with fluorophore-labelled secondary antibodies and fluorescent enhancer. Specifically IFN- $\gamma$-, IL-2- and IFNY\&IL-2-secreting $T$ cells were counted by an automated fluorescence plate reader (AID-iSpot, Straßberg, Germany).

The cutoff of ESAT-6/CFP-10-fluorospot in the differential diagnosis of ATB and LTBI was defined according to our previous research results. The frequency of single IFN- $\gamma$-secreting T cells was 25 (SFCs/2.5 × $10^{5}$ PBMCs), and the proportion of single IFN- $\gamma$-secreting T cells was 43.6\% [15].

\section{Data analysis}

Statistical analysis was performed using the SPSS 24.0 software. The Kolmogorov-Smirnov test was adopted to examine whether the variable data followed a normal distribution. The variables that were normally distributed were denoted as the mean \pm standard deviation (SD), whereas the variables with an abnormal distribution were denoted as the median and IQR. The enumeration data are presented as percentages and $95 \%$ confidence intervals (95\% Cls). The frequency of $\mathrm{T}$ cells between the two groups was compared using two independent sample rank sum tests.

The receiver operating characteristic (ROC) curves of the frequency of IL-2-, IFN- $\gamma^{-}$, IL-2\&IFN- $y$-secreting T cells stimulated by Rv1733c and Rv1733c SLP were developed and the areas under the ROC curves (AUROC) were calculated and compared. The subset of T cells with the largest AUROC was selected as the potential diagnostic marker for distinguishing ATB from LTBI. The optimal cutoff values of the frequency and proportion were determined according to ROC curve analysis. Sensitivity, specificity, positive predictive value (PPV), negative predictive value (NPV), positive likelihood ratio (PLR) and negative likelihood ratio (NLR) were calculated.

The level of significance was defined at $\mathrm{P}<0.05$.

\section{Results}

Demographic and clinical characteristics 
A total of 20 pathogen-confirmed TB and 28 LTBI cases were included. Among them, 18 patients (90\%) were pulmonary TB and 2 patients (10\%) were pulmonary TB with intestinal TB. No significant difference was noted in the parameters gender and age between the two groups. The demographic and clinical features of the participants are shown in Table 1.

Table 1

Demographic and characteristics of the study subjects.

\begin{tabular}{|lll|}
\hline Characteristics & ATB & LTBI \\
\cline { 2 - 3 } & $(\mathbf{n = 2 0})$ & $(\mathbf{n = 2 8 )}$ \\
\hline Age (Median, IQR) & $46(29-62)$ & $47(38-57)$ \\
\hline GENDER & & \\
\hline Male & $12(60 \%)$ & $15(54 \%)$ \\
\hline Female & $8(40 \%)$ & $13(46 \%)$ \\
\hline SITE & & \\
\hline Lung TB & $18(90 \%)$ & $/$ \\
\hline Lung TB and intestinal TB & $2(10 \%)$ & $/$ \\
\hline METHOD & & \\
\hline Acid-fast stain positive & $1(5 \%)$ & $/$ \\
\hline Culture positive & $19(95 \%)$ & $/$ \\
\hline Nucleic acid positive & 0 & $/$ \\
\hline Xpert MTB/RIF positive & 0 & $/$ \\
\hline
\end{tabular}

Comparison of frequencies of MTB IFN- $\gamma$ - and IL-2-secreting T cells in each group

Following stimulation with the Mycobacterium tuberculosis latency-associated antigen Rv1733c SLP, the frequencies of single and total IL-2-secreting T-cells were all higher in the LTBI group than those noted in the ATB group $(P<0.05)$. Following stimulation with Rv1733c, no significant differences were noted in the frequencies of IL-2-, IFN- - - and IFN-ץ\&IL-2-secreting T cells between the ATB and LTBI groups (Fig. 1).

Following stimulation by ESAT- 6 and CFP-10, the frequency and proportion of single IFN- $y$-secreting T cells were significantly higher in the ATB group than those noted in the LTBI group $(P<0.05)$. By contrast, the frequency and proportion of single IL-2-secreting T cells were both significantly lower in the ATB group than those noted in the LTBI group $(P<0.05)($ Table 2$)$. 
Table 2

The frequency and proportion of ESAT-6- and CFP-10-specific single IFN- $y$-, single IL-2-, and dual IFN- $/$ /L2-secreting T cells in patients with ATB and LTBI (iSFCs/250,000 PBMC).

\begin{tabular}{|c|c|c|c|c|c|}
\hline Antigens & Parameter & $\begin{array}{l}\text { Cytokines (Median, } \\
\text { IQR) }\end{array}$ & ATB & LTBI & $\begin{array}{l}P \\
\text { value }\end{array}$ \\
\hline \multirow{6}{*}{$\begin{array}{l}\text { ESAT-6\&CFP- } \\
10\end{array}$} & \multirow[t]{3}{*}{ Frequency } & Single-IL-2 & $5[2.25-10.75]$ & $13[5.5-25.5]$ & 0.012 \\
\hline & & Single-IFN- $\gamma$ & $80[24-212]$ & $20[8-52]$ & 0.015 \\
\hline & & Dual IFN-y/IL-2 & $12[4.5-47]$ & $\begin{array}{l}23.5[11.25- \\
61.25]\end{array}$ & 0.100 \\
\hline & \multirow[t]{3}{*}{ Proportion } & Single-IL-2 & $\begin{array}{l}3.8 \% \\
{[2.5 \%-15.2 \%]}\end{array}$ & $\begin{array}{l}16.7 \% \\
{[14.2 \%-24.0 \%]}\end{array}$ & 0.002 \\
\hline & & Single-IFN- $y$ & $\begin{array}{l}84.6 \% \\
{[71.4 \%-92.5 \%]}\end{array}$ & $\begin{array}{l}32.7 \% \\
{[24.7 \%-63.4 \%]}\end{array}$ & $\dot{0} .001$ \\
\hline & & Dual IFN-y/IL-2 & $\begin{array}{l}14.2 \% \\
{[4.5 \%-24.6 \%]}\end{array}$ & $\begin{array}{l}44.8 \% \\
{[25.3 \%-52.7 \%]}\end{array}$ & $\hat{0}_{0.001}$ \\
\hline
\end{tabular}

Diagnostic accuracy of the IFN-y/IL-2 FluoroSpot assay of Mycobacterium tuberculosis latencyassociated antigen for distinguishing ATB from LTBI

The ROC curves were drawn by the frequencies of single IL-2-, single IFN- $\gamma$ - and double IFN- $y$ \&IL-2secreting T cell stimulation with Rv1733c and Rv1733c SLP. The AUROC, which was $0.711(95 \% \mathrm{Cl}$, $0.566-0.856$ ), drawn by the frequency of single IL-2-secreting T cells stimulated by Rv1733c SLP, was the largest. When the cutoff was $0 \mathrm{SFCs} / 2.5 \times 10^{5}$ PBMCs, the sensitivity and specificity of the differential diagnosis of ATB and LTBI were 75\% (95\% Cl 50.9-91.3\%) and 60.7\% (95\% Cl 40.6-78.5\%), respectively (Fig. 2).

Following stimulation by the MTB-specific antigens ESAT- 6 and CFP-10 peptide, the sensitivity and specificity of the frequency of single IFN- $\gamma$-secreting T cells for the differential diagnosis of ATB and LTBI were $70 \%$ (95\% $\mathrm{Cl} 45.7-88.1 \%)$ and $64.3 \%$ (95\% $\mathrm{Cl} 44.1-81.4 \%)$, respectively. The sensitivity and specificity of the proportion of single IFN-y-secreting T cells were $85 \%(95 \% \mathrm{Cl} 62.1-96.8 \%)$ and $71.4 \%$ (95\% Cl 51.3-86.8\%) (Table 3). 
Table 3

Diagnostic value of Fluorospot method to differentiate ATB and LTBI when stimulated by ESAT-6/CFP-10 and Rv1733c SLP

\begin{tabular}{|c|c|c|c|c|c|c|}
\hline & $\begin{array}{l}\text { Sensitivity } \\
(\%, 95 \% \mathrm{Cl})\end{array}$ & $\begin{array}{l}\text { Specificity } \\
(\%, 95 \% \mathrm{Cl})\end{array}$ & $\begin{array}{l}\text { PLR (\%, } \\
95 \% \mathrm{Cl})\end{array}$ & $\begin{array}{l}\text { NLR (\%, } \\
95 \% \mathrm{Cl})\end{array}$ & $\begin{array}{l}\text { PPV } \\
\text { (95\% Cl) }\end{array}$ & $\begin{array}{l}\text { NPV } \\
(95 \% \mathrm{Cl})\end{array}$ \\
\hline $\begin{array}{l}\text { Frequencies } \\
\text { of single } \\
\text { IFN-Y- } \\
\text { secreting T } \\
\text { cells } \\
\text { stimulated } \\
\text { by EAST-6\& } \\
\text { CFP-10 }\end{array}$ & $\begin{array}{l}70.0(45.7- \\
88.1)\end{array}$ & $\begin{array}{l}64.3(44.1- \\
81.4)\end{array}$ & $\begin{array}{l}1.96(1.10- \\
3.48)\end{array}$ & $\begin{array}{l}0.47(0.23- \\
0.96)\end{array}$ & $\begin{array}{l}58.3(36.6- \\
77.9)\end{array}$ & $\begin{array}{l}75.0(53.3- \\
90.2)\end{array}$ \\
\hline $\begin{array}{l}\text { Proportion } \\
\text { of single } \\
\text { IFN- } \gamma^{-} \\
\text {secreting T } \\
\text { cells } \\
\text { stimulated } \\
\text { by EAST-6\& } \\
\text { CFP-10 }\end{array}$ & $\begin{array}{l}85.0(62.1- \\
96.8)\end{array}$ & $\begin{array}{l}71.4(51.3- \\
86.8)\end{array}$ & $\begin{array}{l}2.98(1.61- \\
5.50)\end{array}$ & $\begin{array}{l}0.21(0.07- \\
0.61)\end{array}$ & $\begin{array}{l}68.0(46.5- \\
85.1)\end{array}$ & $\begin{array}{l}86.96(66.4- \\
97.2)\end{array}$ \\
\hline $\begin{array}{l}\text { Frequencies } \\
\text { of single IL- } \\
\text { 2-secreting } \\
\text { T cells } \\
\text { stimulated } \\
\text { by Rv1733c } \\
\text { SLP }\end{array}$ & $\begin{array}{l}75.0(50.9- \\
91.3)\end{array}$ & $\begin{array}{l}60.7(40.6- \\
78.5)\end{array}$ & $\begin{array}{l}1.91(1.13- \\
3.23)\end{array}$ & $\begin{array}{l}0.41(0.18- \\
0.93)\end{array}$ & $\begin{array}{l}57.6(36.0- \\
76.7)\end{array}$ & $\begin{array}{l}77.3(54.6- \\
92.2)\end{array}$ \\
\hline Parallel test & $\begin{array}{l}100.0(83.2- \\
100.0)\end{array}$ & $\begin{array}{l}35.7(18.6- \\
55.9)\end{array}$ & $\begin{array}{l}1.56(1.18- \\
2.05)\end{array}$ & 0 & $\begin{array}{l}52.6(35.8- \\
69.0)\end{array}$ & $\begin{array}{l}100.0(69.2- \\
100.0)\end{array}$ \\
\hline Serial test & $\begin{array}{l}45.0(23.1- \\
68.5)\end{array}$ & $\begin{array}{l}92.9(76.5- \\
99.1)\end{array}$ & $\begin{array}{l}6.30(1.52- \\
26.08)\end{array}$ & $\begin{array}{l}0.59(0.39- \\
0.89)\end{array}$ & $\begin{array}{l}81.8(48.2- \\
97.7)\end{array}$ & $\begin{array}{l}70.3(53.0- \\
84.1)\end{array}$ \\
\hline
\end{tabular}

Diagnostic accuracy of multiple tests

The assessment of the frequency and proportion of single IFN- $y$-secreting T cells stimulated by MTBspecific antigen ESAT-6\&CFP-10 was combined by the frequency of single IL-2-secreting T cells stimulated by Rv1733c SLP. The parallel test increased the sensitivity to $100 \%$ (95\% Cl, 83.16-100.00\%), the negative likelihood ratio to 0 and the negative predictive value to $100 \%(95 \% \mathrm{Cl}, 69.15-100.00 \%)$. Moreover, the serial test increased the specificity to $92.86 \%$ (95\% Cl, 76.50-99.12\%), the positive likelihood ratio to $6.3(95 \% \mathrm{Cl}, 1.52-26.08)$ and the positive predictive value to $81.82 \%(95 \% \mathrm{Cl}, 48.22-97.72 \%)$ (Table 3).

\section{Discussion}

The present study conducted a preliminary investigation of the diagnostic value of Rv1733c/Rv1733c SLP, the mycobacterium tuberculosis latency-associated antigen, combined with the EAST-6/CFP-10- 
Fluorospot for the differential diagnosis of ATB and LTBI.

During LTBI, Mtb is contained within granulomas and is composed mainly of activated macrophages. It recruits inflammatory cells and can be isolated from the infected cells in an organized structure. In this way, it creates an environment associated with containment of Mtb infection, dissemination and multiplication. The immune response inside the granuloma generates a set of particular conditions that include oxygen deprivation, low pH and nutrient starvation, in which Mtb is able to enter a defined nonreplicating state $[15,16]$. Several in vitro models have shown that Mtb is capable of an extensive repertoire of metabolic realignments that can cause its adaptation to the variety of environmental stresses. However, when the host's immunity or the signals maintaining granuloma structure are reduced, dormant Mtb is activated, which proliferates and disseminates, eventually developing into active tuberculosis $[17,18]$. Although bacterial and host factors that induce and maintain latent M. tuberculosis infection are ill defined, recent studies have shown that during the dormancy of Mtb, the expression of dormancy survival regulon (DosR) is upregulated. Through the interaction of dormancy-associated proteins with macrophages, the formatting and maintenance of the granuloma structure is required to allow Mtb to transit into a stage of dormancy [19]. It is worth noting that the immune response to the Dos $R$ regulon encoded antigens is associated with the containment during latent phases of $M$. tuberculosis infection, since the antigens encoded by several DosR regulons can be preferentially recognized by the $T$ cells of $L T B I$ subjects, in order to induce the activation of $T$ cells associated with the control of Mtb infection. This process in turn produces various cytokines, such as IFN- $y$, TNF and IL-2, preventing the progression of ATB $[20,21]$. Similarly, following injection into the body as a preventive vaccine, the DosR regulon encoded antigens can reduce the Mycobacterium tuberculosis infection in the lung [22]. Therefore, the immune response against these antigens may contribute in controlling latent $\mathrm{M}$. tuberculosis infection and preventing reactivation of tuberculosis [23,24]. Previous studies have shown that while responsiveness to early secretory antigenic target 6 is an optimal marker of M. tuberculosis infection, a strong response to the dormant antigen is largely restricted to latently infected individuals, offering the possibility of differential immunodiagnosis or therapeutic vaccination against TB [25].

Leyten et al. [20] stimulated human PBMCs with 25 types of proteins expressed by DosR regulators. The results indicated that Rv1733c-stimulated LTBI subjects produced higher levels of IFN-y compared to those of ATB patients. The present study demonstrated that the levels of IFN- $\gamma$ produced by Rv1733c in the LTBI group were almost equal with those of the ATB group, which was different from Leyten's results. Furthermore, the results of the present study indicated that the levels of IL-2 produced by $T$ cell stimulation with Rv1733c in the LTBI group were higher than those of the ATB group. In addition, these findings may be attributed to the degree of immune response required for stimulation by Rv1733c being too weak or the small sample size, which resulted in no significant differences between the two groups.

In accordance with the results of Mariateresa Coppola et al. [12], the present study demonstrated that Rv1733c SLP could be well recognized by T cells of LTBI subjects and produced higher levels of IFN- $Y$ than Rv1733c. Therefore, Rv1733c SLP exhibited stronger immunogenicity and protection ability. Consistent with the findings of Abebech Demissie et al. [25], the latency-associated antigen (Rv2031c) 
mainly induced a strong immune response in LTBI individuals, which could be used for differential immune-diagnosis of tuberculosis. In the present study, the frequency of T cells following stimulation with Rv1733c SLP in the ATB and LTBI groups confirmed that Rv1733c SLP induced higher number of T cells to produce higher levels of cytokines in the LTBI group, while the difference in the frequency of single IL-2-secreting $T$ cells was significant between the two groups. Therefore, the latency associated antigen Rv1733c SLP may be possibly used to distinguish between ATB and LTBI.

The results of the ESAT-6/CFP-10-Fluorospot indicated that the frequency and proportion of single IFN-ysecreting T cells in the ATB group were significantly higher than those in the LTBI group $(P<0.05)$, whereas the frequency and proportion of single II-2-secreting T cells in the ATB group were significantly lower than those in the LTBI group $(P<0.05)$. This is consistent with our previous results [13]. On this basis, the immune response against Rv1733c SLP could improve the diagnosis of latent infection.

Clinical diagnosis is a dynamic process. The pretest probability of the disease can be estimated by clinical analysis based on the comprehensive medical history, physical examination and laboratory results [26]. A certain diagnostic test is performed on the basis of the pretest probability, which may increase or decrease the possibility of initial diagnosis. The disease probability at that time is called the posttest probability. The estimation of the LR [27] of a diagnostic test can distinguish the posttest probability from the pretest probability, reflecting the extent to which the results of a diagnostic test will increase or decrease the pretest probability of the target disease [28]. The data of the current study indicated that the combination of the ESAT-6/CFP-10-fluorospot with the Rv1733c SLP resulted in an increase in the positive likelihood ratio from 3.64 to 6.3 and in the positive predictive value from $72.22-$ $81.82 \%$ when the serial test was used. In contrast to these findings, the parallel test decreased the negative likelihood ratio from 0.17 to 0 and increased the negative predictive value from $88.89-100 \%$. Rv1733c SLP combined with ESAT-6/CFP-10-fluorospot can improve the accuracy of differential diagnosis between ATB and LTBI subjects. Therefore, parallel or sequence tests can be selected to improve sensitivity or specificity, respectively in order to aid the exclusion or diagnosis of ATB.

The advantages of the present study can be highlighted as follows: 1) The representative M. tuberculosis latency associated antigens Rv1733c and Rv1733c SLP were added on the basis of the M. tuberculosis specific antigens ESAT-6 and CFP-10; 2) The IFN- - /IL-2 Fluorospot method was used to simultaneously detect the secretion of IFN-y and IL-2 cytokines at the single cell level, saving manpower and blood samples to the greatest extent. The limitations of the present study can be summarized as follows: 1 ) The small sample size and large confidence intervals, which decrease the accuracy of the results; 2) The case-control study design and the fact that all ATB patients were pathogen-conformed, which may amplify the diagnostic accuracy. The accuracy of the differential diagnosis of ATB and LTBI requires further prospective cohort studies for confirmation.

\section{Conclusion}


Rv1733c SLP can be used as an alternative antigen for the TB diagnosis test based on the T-cell immune reaction. On the basis of the Mtb specific antigens ESAT- 6 and CFP-10, the combination with Rv1733c SLP is helpful for the differential diagnosis of ATB and LTBI. This conclusion needs to be verified by prospective studies with a larger sample size.

\section{Declarations}

\section{Acknowledgements}

Not applicable.

\section{Authors' Contributions}

This study was designed and conducted by LXQ. ZLF supervised this study; HMM collected data, carried out the analysis and wrote the manuscript; WSJ and ZYQ participated in laboratory works.GMQ and LXQ collected patients; ZLF amended the manuscript. All authors read and approved the final manuscript.

\section{Funding}

This study was funded by the National Science and Technology Major Project of the People's Republic of China (grant number 2017ZX10201302); Chinese Academy of Medical Sciences Initiative for Innovative Medicine (grant number 2016-I2M-1-013, 2019-I2M-2-005).

\section{Availability of data and materials}

Not applicable.

\section{Ethics approval and consent to participate}

The present study was conducted in accordance with the Declaration of Helsinki and was approved by the Ethics Committee of PUMCH. Written informed consent was obtained from all patients prior to their enrollment in this study. We confirmed that the identification information of all participants (including patient names, ID numbers, home addresses and telephone numbers) would not be included in recordings, written descriptions or publications.

\section{Consent for publication}

Not applicable.

\section{Competing interests}

All authors declare that they have no competing interest.

\section{Author details}


${ }^{1}$ Department of Infectious Diseases, Peking Union Medical College Hospital, Chinese Academy of Medical Sciences and Peking Union Medical College, Beijing 100730, China. ${ }^{2}$ Center for Tuberculosis Research, Chinese Academy of Medical Sciences and Peking Union Medical College, Beijing 100730, China.

${ }^{3}$ Clinical Epidemiology Unit, Peking Union Medical College, International Clinical Epidemiology Network, Beijing 100730, China. ${ }^{4} \mathrm{PUMCH}$ 's Center for the Research of Translational Medicine, Beijing 100730, China. ${ }^{5}$ Department of Tuberculosis, Beijing Chest Hospital, Capital Medical University/Beijing Tuberculosis and Thoracic Tumor Research Institute, Beijing, P.R. China.

\section{References}

1. WHO Global tuberculosis report 2020. http://www.who.int/tb/publications/global_report/en/.

2. Zhang, L., et al., Utility of T-cell interferon-y release assays for diagnosing tuberculous serositis: a prospective study in Beijing, China. PLoS One, 2014. 9(1): p. e85030.

3. Behr, M.A., et al., Comparative genomics of BCG vaccines by whole-genome DNA microarray. Science, 1999. 284(5419): p. 1520-3.

4. Zhang, X., et al., The immune characterization of interferon- $\beta$ responses in tuberculosis patients. Microbiol Immunol, 2018. 62(4): p. 281-290.

5. Pai, M., et al., Gamma interferon release assays for detection of Mycobacterium tuberculosis infection. Clin Microbiol Rev, 2014. 27(1): p. 3-20.

6. Menzies, D., M. Pai, and G. Comstock, Meta-analysis: new tests for the diagnosis of latent tuberculosis infection: areas of uncertainty and recommendations for research. Ann Intern Med, 2007. 146(5): p. 340-54.

7. Voskuil, M.I., Mycobacterium tuberculosis gene expression during environmental conditions associated with latency. Tuberculosis (Edinb), 2004. 84(3-4): p. 138-43.

8. Black, G.F., et al., Immunogenicity of novel DosR regulon-encoded candidate antigens of Mycobacterium tuberculosis in three high-burden populations in Africa. Clin Vaccine Immunol, 2009. 16(8): p. 1203-12.

9. Esmail, H., C.E. Barry, 3rd, and R.J. Wilkinson, Understanding latent tuberculosis: the key to improved diagnostic and novel treatment strategies. Drug Discov Today, 2012. 17(9-10): p. 514-21.

10. Sutherland, J.S., et al., Analysis of host responses to Mycobacterium tuberculosis antigens in a multisite study of subjects with different TB and HIV infection states in sub-Saharan Africa. PLoS One, 2013. 8(9): p. e74080.

11. Reece, S.T., et al., Improved long-term protection against Mycobacterium tuberculosis Beijing/W in mice after intra-dermal inoculation of recombinant BCG expressing latency associated antigens. Vaccine, 2011. 29(47): p. 8740-4.

12. Coppola, M., et al., Synthetic Long Peptide Derived from Mycobacterium tuberculosis Latency Antigen Rv1733c Protects against Tuberculosis. Clin Vaccine Immunol, 2015. 22(9): p. 1060-9. 
13. Zhang, L., et al., Utility of Th1-cell immune responses for distinguishing active tuberculosis from nonactive tuberculosis: A case-control study. PLoS One, 2017. 12(5): p. e0177850.

14. Lifan, Z., et al., Application of IFN-Y/IL-2 FluoroSpot assay for distinguishing active tuberculosis from non-active tuberculosis: A cohort study. \% J Clinica chimica acta; international journal of clinical chemistry. 2019. 499.

15. Gupta, A., et al., Mycobacterium tuberculosis : Immune evasion, latency and reactivation \% J Immunobiology. 2012. 217(3).

16. Saunders, B.M. and W.J. Britton, Life and death in the granuloma: immunopathology of tuberculosis. Immunol Cell Biol, 2007. 85(2): p. 103-11.

17. Kondratieva, T., et al., Latent tuberculosis infection: what we know about its genetic control? Tuberculosis (Edinb), 2014. 94(5): p. 462-8.

18. Gengenbacher, M. and S.H. Kaufmann, Mycobacterium tuberculosis: success through dormancy. FEMS Microbiol Rev, 2012. 36(3): p. 514-32.

19. Serra-Vidal, M.M., et al., Immunogenicity of 60 novel latency-related antigens of Mycobacterium tuberculosis. Front Microbiol, 2014. 5: p. 517.

20. Leyten, E.M., et al., Human T-cell responses to 25 novel antigens encoded by genes of the dormancy regulon of Mycobacterium tuberculosis. Microbes Infect, 2006. 8(8): p. 2052-60.

21. Roupie, V., et al., Immunogenicity of eight dormancy regulon-encoded proteins of Mycobacterium tuberculosis in DNA-vaccinated and tuberculosis-infected mice. Infect Immun, 2007. 75(2): p. 941-9.

22. Kaufmann, S.H., How can immunology contribute to the control of tuberculosis? Nat Rev Immunol, 2001. 1(1): p. 20-30.

23. Commandeur, S., et al., Double- and monofunctional CD4(+) and CD8(+) T-cell responses to Mycobacterium tuberculosis DosR antigens and peptides in long-term latently infected individuals. Eur J Immunol, 2011. 41(10): p. 2925-36.

24. Riano, F., et al., $T$ cell responses to DosR and Rpf proteins in actively and latently infected individuals from Colombia. Tuberculosis (Edinb), 2012. 92(2): p. 148-59.

25. Demissie, A., et al., Recognition of stage-specific mycobacterial antigens differentiates between acute and latent infections with Mycobacterium tuberculosis. Clin Vaccine Immunol, 2006. 13(2): p. 179-86.

26. Richardson, W.S.J.E.B.M., Where do pretest probabilities come from. 1999. 4(3): p. 68-.

27. Grimes, D.A. and K.F. Schulz, Refining clinical diagnosis with likelihood ratios. Lancet, 2005. 365(9469): p. 1500-5.

28. Sackett, D.L., The rational clinical examination. A primer on the precision and accuracy of the clinical examination. Jama, 1992. 267(19): p. 2638-44.

\section{Figures}



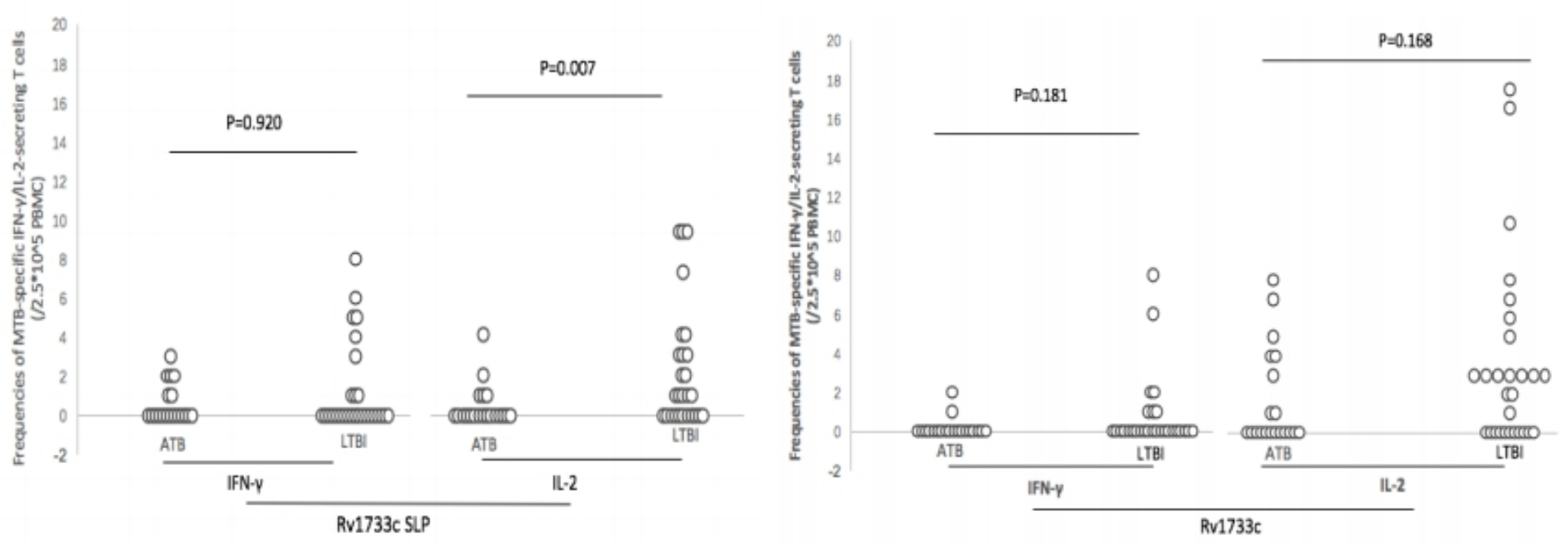

\section{Figure 1}

Comparison the frequencies of IFN-y/ IL-2-secreting T cells stimulated by Rv1733c SLP, Rv1733c between ATB and LTBI. ATB=active tuberculosis $(n=20)$. LTBI=latent tuberculosis infection $(n=28)$.
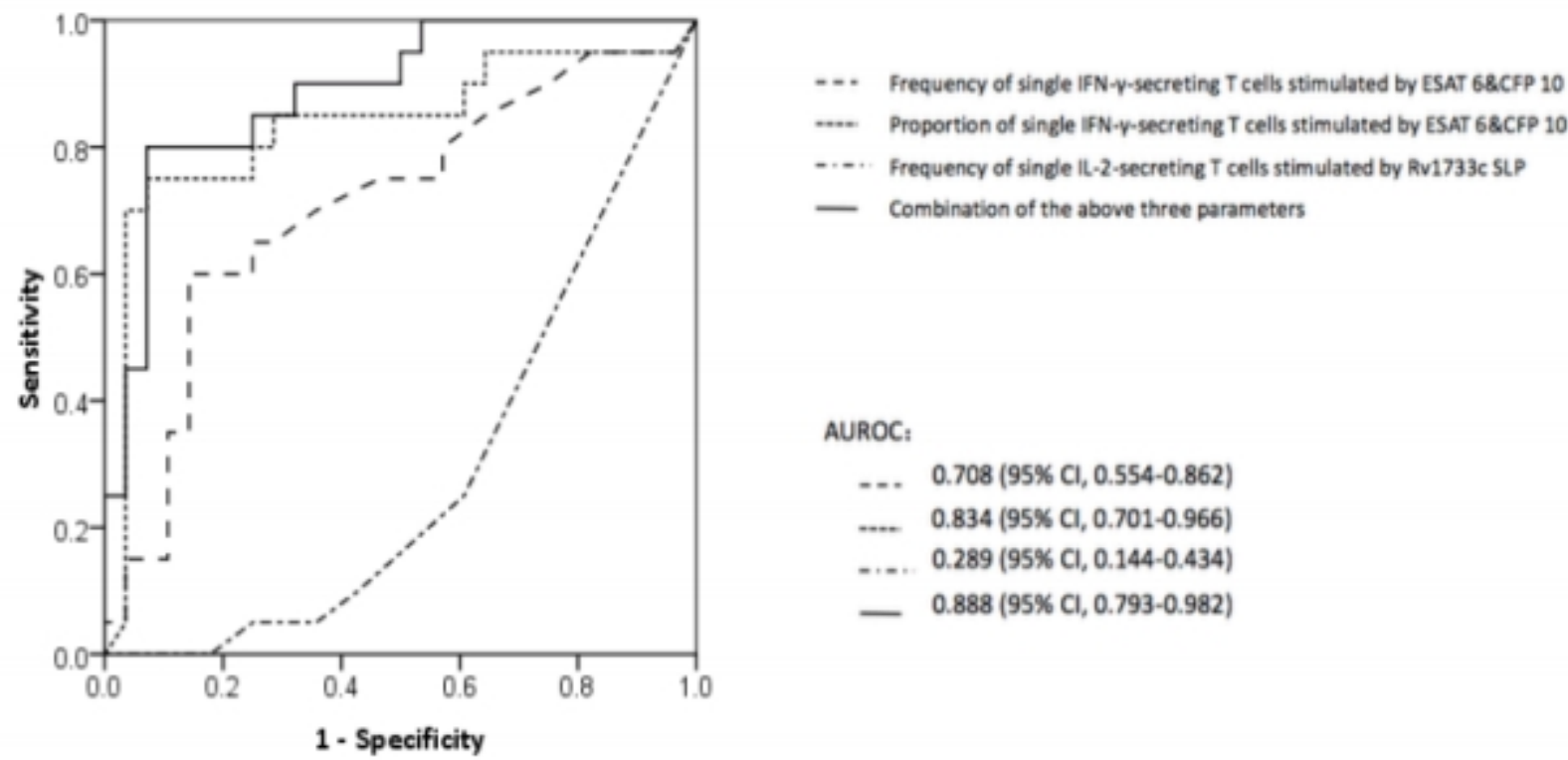

AUROC:

-.. $0.708(95 \% \mathrm{Cl}, 0.554-0.862)$

$0.834(95 \% \mathrm{Cl}, 0.701-0.966)$

$0.289(95 \% \mathrm{Cl}, 0.144-0.434)$

$-0.888(95 \% \mathrm{Cl}, 0.793-0.982)$

Figure 2

ROC curve for differentiating ATB from LTBI. Supplementary table 1. The frequencies of Rv1733c SLP-, Rv1733c-specific single IFN- $\gamma$-, single IL-2-, total IFN- $\gamma$-, total IL-2-, and dual IFN- $\gamma /$ IL-2-secreting T cells in patients with ATB and LTBI (iSFCs/250,000 PBMC).

\section{Supplementary Files}

This is a list of supplementary files associated with this preprint. Click to download.

- Supplementarytable1.docx 